\title{
Contents, Vol. 28, 1912
}

\section{Inhalts - Verzeichnis.}

Seito

Original-Arbeiten.

Attias, G., Arcus juvenilis und Arcus senilis corneae .... 539 Augstein, K., Bemerkungen zur

Farbensinnuntersuchung . . . 3475/8 Birch-Hirschfeld, A., Zum fKapitel der Sonnenblendung des Auges. (Hierzu Ta. V-VI) 324, 444, 509

Bistis, J., Ueber die nach Arsenobenzol auftretenden Augen-

komplikationen und ihre Bedeutung . 150

Blaauiv, Edmond, Ein Fall von Cysten im oberen Fornix . . 50 Dalmer, Max, Beitrag zur

Ophthalmia nodosa 356

Dutoit, A., Versuche mit interner Jodtherapie bei Glaukom 131 Handmann, M., Ueber temporäre Myopie bei orbitalen Neu-

bildungen $\quad 542$

Horovitz, J., Der Einfluß von Kokain und Homatropin auf Ak-

kommodation und Pupillengröße $\quad 530$

Junius, P.. Dystrophia margin alis corneae bei einem jugend-

lichen Mamie (Hierzu Taf. I) 43

. Zellstudien bei Trachom. (Hierzu Taf. VII-VIII) . 409

Kratiß, W., Ueber eine noch nicht am Augenlid beobachtete

Geschwulst: Ganglioneurom. (Hierzu Taf. II-III) . .110 Marenholtz, v., Beiträge zu den

Erkrankungen der Orbita . . 53 -. Ein Beitrag zu den Blitzschädigungen des Auges . . 449 -. Ein

Beitrag zur Aetiologie, Pathologie und Therapie

des Pemphigus conjunctivae 550

Reis. W., Ueber Ringsarkom des Ciliarkörpers. (Hierzu

Taf. IX-X) 426

Schieck, F., Die Anwendung der Ergebnisse der Inimunitäts-

forschung auf die Augenheilkunde 24

Stargardt, K., und L. Olojf, Ueber die Bedeutung und die

Methodik der Farbensinnuntersuchung 1

Stimmel, F. und F. Rotter, Beitrag zur Pathologie und Therapie

des Hydrophthalmus congenitus. (Hierzu Taf. IV) . . 114 Toczyski, Franz, Ueber den Einfluß des Dionins auf das Ver-

halten der Pupille und der Tension normaler Augen . : 32

$-\mathrm{IV}-$

Seite

Voirol, August Friedrich, Untersxichungen über Refraktion, Visus, Farbensinn xmd

Muskelgleichgewicht aix denAxxgen von 939 Schulkindern

95

Wolff, Hugo, Korrektion der Anisometropsie bei Aphakie 
mittels eixxes Zeiß"scheix zweigliedrigen Linsensystems 149 --, Ueber die zentrische

13/4ílexlose Mikro-Ophthalmoskopie 307

Berichte über die deutsche ophthalmologieche Liteialur.

Diagnostik xxxid Extraktion von Fremdkörpern (1911). Von

Dr. Asmus ixx Düsseldorf 59

Anatomie des Axxges (II. Sem. 1911). Von Prof. Dr. Sobotta

in Würzbxxrg • 157

Die angeborenen Anomalien xuxd Mißbildungen des Axxges(I. Semester 1912). Kritisches

Sammelreferat. erstattetvon Priv.-Doz. Dr. B. Seefelder in Leipzig 173

Bericht über die Mikroorganismen (I. Semester 1912). Von

Priv.-Doz. Dr. Zade in Heidelberg 182

Physiologic des G·esiehtssiniies xxnd des Bewegxxixgsappa-rates (I. Halbjalxr 1912). Von

Privatdozent Dr. Köllnerm Berliix 360

Spezielle Pathologic xxixd TJxerapie (I. xxnd II. Semester 1911).

Referent: Dr. Konrad Schroder iix Gera . . . 371. 472.556

Dioptrik xxnd Anomalien der Refraktion xxnd Akkommodation.Von Prof. Dr. Maximilian

Salzmann (II. Semester 1911xxnd I. Semester 1912) 466

Untersuchungsmethoden (I. xxnd II. Semester 1911). Von

Prof. Dr. F. Dimmer ixx Wieix 553

Berichte über die ausländisehe ophthalmologieche Literatur.

Bericht über die exxglisclxe ophthalmologisehe Literatxxr

(I. Semester 1912). Von. Dr. Ch. Markus in Londoix . . 452

Bericht über die íraxazösische Literatxxr (I. Senxester 1911).

Von Dr. Haeffner in Wiesbadexx 187

Bericht über die holländische ophthalmologisehe Literatur des Jahres 1912 (I. Semester). Von

Dr. G. J. Schoute in Amsterdanx xxtxd Prof. Dr. W. Koster-Gzn in Leidexx . . 238

Bericht über die italienische Literatur. Von Dr. Speciale-

Cirincione in Rom. $1910 \quad 215$

$-191137^{*}$

Bericht über die polnische ophthalmologisehe Literatxxr im

Jahre 1911. Erstattet voix Dr. V. Kamocki in Warschaxx 200

Bericht über die skaixdiixavische ophthalmologisehe Literatxxr(I. und II. Semester 1910). Yon

Priv.-Doz. Dr. ErnstForsmark in Stockholm 65

$-\mathrm{V}-$

Seite

Gesellschaftsberichte.

Bericht über die XXXVIII. Zusammenkunft der Ophthalmo-logischen Gesellschaft in Heidelberg

vom 3.-5. August1912. Unter Benutzung von Autoreferaten erstattet vonDr. P. Junius in Köln 245

Bericht über die Sitzung der Abteilung iür Augenheilkunde der84. Versammlung deutscher

Naturforscher und Aerzte inMünster i. W. am 16. September 1912490

Bericht über die VIII. Versammlung der ungarischen ophthal-mologischen Gesellschaft in

Budapest. Erstattet vonDoz. Dr. L. von Bïaskovics in Budapest 278

Berliner ophthalmologische Gesellschaft. Sitzungen vom

13. Juni. 17. Juli und 17. Oktober 1912 . . . 289. 392, 604

Verein der Augenärzte von Ost- und Westpreußen. Sitzung 
am 9. Juni 1912

286

Ophthalmologische Gesellschaft in Wien. Sitzungen vom

27.November und 18. Dezember 1911 und 12.Februar 1912596

St. Petersburger ophthalmologische Gesellschaft. Sitzungen

am 23. Februar, 19. April und 27. September 1912 . 393. 593

Unfall- und Versicherungskuntie .... 80, 292, 395, 493. 607

Diagnostische Notizen $\quad 81,297,396,496.610$

Therapeutisehe Umschau $\quad 83,298,399,500,612$

Nase und Auge $\quad 78$

Buchanzeigen 503

Literatur-Verzeiehnis 88, 302, 403. 504, 615

Offene Korrespondenz 606

Tagesnachrichten 92.306, 508

S. Sachregister $\quad 619$

Namenregister 631 